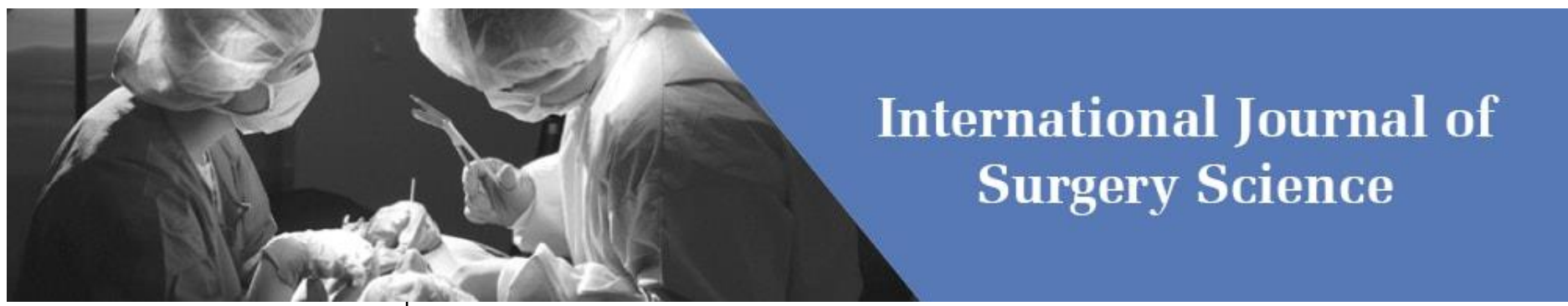

E-ISSN: 2616-3470

P-ISSN: 2616-3462

(C) Surgery Science

www.surgeryscience.com

$2019 ; 3(3): 190-193$

Received: 04-05-2019

Accepted: 06-06-2019

Dr. Sumit Shukla

Prof. Department of General

Surgery, MGM Medical College \&

M.Y. Hospital, Indore,

Madhya Pradesh, India

Dr. Shahrukh Memon

Resident of Surgery, Department of General Surgery, MGM Medical College \& M.Y. Hospital, Indore,

Madhya Pradesh, India

Correspondence

Dr. Sumit Shukla

Prof. Department of General

Surgery, MGM Medical College \&

M.Y. Hospital, Indore,

Madhya Pradesh, India

\section{Study on management of blunt trauma abdomen with special reference to clinical and radiological factors affecting decision making in emergency}

\author{
Dr. Sumit Shukla and Dr. Shahrukh Memon
}

DOI: https://doi.org/10.33545/surgery.2019.v3.i3d.167

\section{Abstract}

Background: All Blunt Trauma Abdomen with special reference to Clinical and Radiological factors affecting decision making in Emergency of above $14 \mathrm{yr}$ age group by blunt trauma admitted in routine \& emergency hours in General Surgery in MGM Medical College \& M.Y. Hospital, Indore.

Result: In this study maximum no. of patients were in age group between 26 to 50 (56), followed by 12 to 25 (37) \& in 51 to 75 (07), there were 84 males \& 16 females in our study. In our study maximum Mode of Trauma were in RTA (73), Assault (16), others (04), fall from Height (03) \& Falling blunt object over body (02). Maximum Extra Abdominal Solid Organ Injury were seen in No External Injury (54), followed by facial injury, chest and facial injury, head injury i.e. (02). Maximum Complications were seen in Wound infection (05), Septicemia (03), Bed Sore (03), ARF (03). Management of Blunt Trauma Abdomen highest cases were seen in Liver (57), Spleen (44), Kidney (16) \& Pancreases (09).

Conclusion: In Blunt Trauma Abdomen injury there was maximum incidence of liver injury (57), followed by spleen injury (47), followed by renal injury (16) and followed by pancreatic injury cases (09) involved in BAT Blunt Trauma Abdomen organ injury grading \& management according to AAST guideline (based on CT finding). In our study we tried to develop the management protocol for patients with clinical examination and findings.

Keywords: Blunt trauma abdomen, clinical, radiological \& emergency

\section{Introduction}

Blunt abdominal trauma is quite common since previous time and increasing day by day in emergency clinics. Many of time this injury is life threatening and require urgent treatment.

Blunt abdominal trauma can result in laceration of solid organ usually causing bleeding which in its most severe form manifest as hemorrhagic shock or as visceral perforation of GI tract. Abdominal compartment syndrome is now recognized as a frequent confounder of surgical critical care following blunt abdomen trauma ${ }^{[1]}$.

Trauma is defined as damage to the body caused by an exchange with environmental energy that is beyond the body's resilience Abdomen is a commonly injured body region in blunt trauma abdomen and frequently requires the care of surgeon for definitive management. The vital nature of the organ contained within the abdomen makes evaluation and management a priority. The predominant source of morbidity are bleeding and visceral perforation with associated sepsis ${ }^{[2]}$. In the setting of blunt trauma, solid organs often sustain contusion and laceration, causing bleeding that may require surgical management. Furthermore, blunt forces can cause rupture of hollow viscera due to rapid compression of a segment of intestine containing fluid and air.1 Of all patient in 2016 NTBD sustained mortality rate of $12.87 \%$ was present. Approx 7 patients out of 20 patients in week in M.Y. Hospital, Indore are reported as blunt trauma abdomen and its number is increasing every year ${ }^{[3]}$.

Motor vehicle collisions are a common source of blunt trauma abdomen. Seat belt reduces the chances of head injury and chest injury but posts a threat to abdominal organs like pancreas and intestines. Paediatric age group is vulnerable to abdominal injury by seat belts. Other causes include sports which affects kidneys and spleen, fall (from height or on blunt objects like bicycle handles), assault (blow or kick to abdomen) ${ }^{[4]}$.

The most common solid organ to be injured in abdominal trauma is Spleen and liver, however injuries to pancreas, bowel and mesentery, urinary bladder and kidneys are less common but 
reported. Usually the kidney and bladder injury are associated with pelvic trauma and retroperitoneal haemorrhage. Mostly the in-road traffic accident patient's injury to Head, Chest and bones should be ruled out.

All patients are managed according to Advanced Trauma life Support principles. Chest X-Ray is mandatory. Unstable patients are subjected to e-FAST and free intra-abdominal fluid is identified are subjected to operating room and are explored. All patients who respond to resuscitation are reassessed and selectively investigated with abdominal CT.

The Organ Injury Scaling committee of American Association for Surgery of Trauma (AAST) has developed severity scores for number of organ system. These grading have been scaled from I to VI. We utilised the AAST grading system for management of solid and hollow viscous injuryin our study.

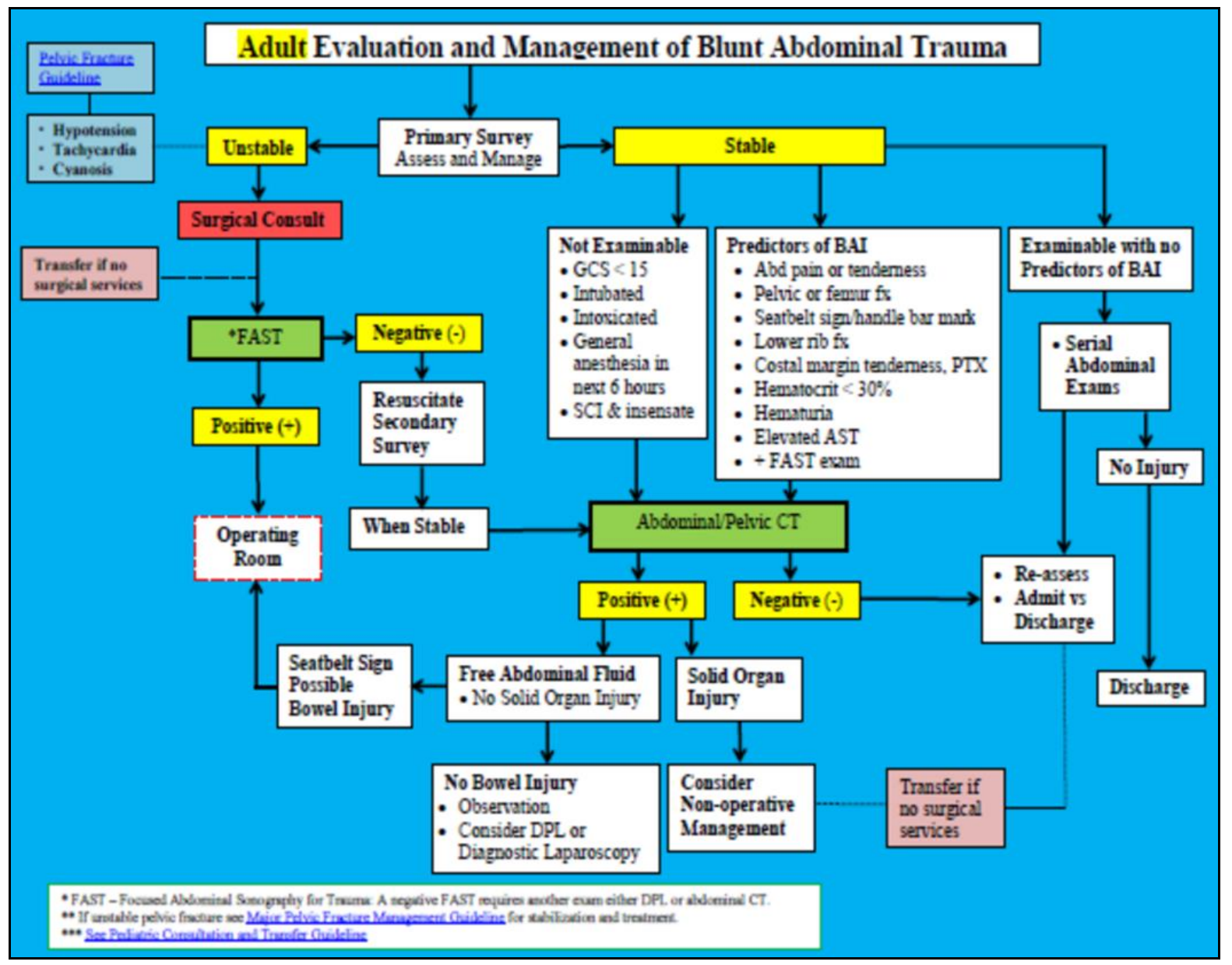

Algorithm for management of blunt trauma abdomen patients

\section{Material \& Method}

All Blunt Trauma Abdomen with special reference to Clinical and Radiological factors affecting decision making in Emergency of above $14 \mathrm{yr}$ age group by blunt trauma admitted in routine \& emergency hours in General Surgery in MGM Medical College \& M.Y. Hospital, Indore, in duration Dec 2017 Dec 2018 with the help of the resident surgical officers looking after the admitted patients as prospective analysis with Blunt Trauma Abdomen.

Special focus on patient Blunt Trauma Abdomen with reference type surgery; conservative or open surgery and outcome of surgery. Diagnostic methods applied and investigation of choice for the diagnosis.

Total 100 cases of Blunt Trauma Abdomen. Mode of Injury will be defined according to history taken from the patient (if conscious) or relatives with history of blunt abdominal trauma causing injury.

\section{Evaluation of Blunt Trauma Abdomen and Management ${ }^{[4]}$}

Etiology and Mechanism of blunt abdominal injuries Causative agent Include patient who sustained injury due to-
- Assault by Fists and blows, Lathy, Iron rods, Kicks

- Received accidental animal kicks over abdomen

- Got injured due to fall of some heavy object like log, gravel, got buried in mine.

- Rolled down the stairs or stumbled over some blunt object.

\section{Inclusion Criteria}

1. All patient of age 20-60 years Blunt Trauma abdomen and associated co morbidities

2. Attenders and patients who give written informed consent.

3. Patients having blunt trauma abdomen only.

\section{Exclusion Criteria}

1. Attender and Patients not willing to give written consent.

2. Patient of age below 20 and above 60 years.

3. Excluding the patients other than blunt trauma abdomen.

\section{Statistical Analysis}

All Statistical data was analysed \& calculated on MS Excel \& SPSS 20.0. 


\section{Results}

Table 1: Age \& Gender Distribution

\begin{tabular}{|c|c|c|}
\hline S. No. & Age Distribution & No. \\
\hline 1 & 12 to 25 & 37 \\
\hline 2 & 26 to 50 & 56 \\
\hline 3 & 51 to 75 & 7 \\
\hline & & No. \\
\hline & Gender Distribution & 16 \\
\hline 1 & Female & 84 \\
\hline 2 & Male & \\
\hline
\end{tabular}

In this study maximum no. of patients were in age group between 26 to 50 (56), followed by 12 to 25 (37) \& in 51 to 75 (07), there were 84 males \& 16 females in our study.

Table 2: Mode of Trauma

\begin{tabular}{|c|c|c|}
\hline S. No. & Mode of trauma & No. \\
\hline 1 & Accidental fall & 2 \\
\hline 2 & Assault & 16 \\
\hline 3 & Fall from height & 3 \\
\hline 4 & Falling blunt object over body & 2 \\
\hline 5 & RTA & 73 \\
\hline 6 & Others & 4 \\
\hline
\end{tabular}

In our study maximum Mode of Trauma were in RTA (73), Assault (16), others (04), fall from Height (03) \& Falling blunt object over body (02).

Table 3: Extra abdominal solid organ injury

\begin{tabular}{|c|c|c|}
\hline S. No. & Extra abdominal solid organ injury & No. \\
\hline 1 & facial injury & 2 \\
\hline 2 & \# L3 vertebrae and lower extremity \# & 1 \\
\hline 3 & $10^{*} 0.5 \mathrm{cms}$ bruise in infraumblical region & 1 \\
\hline 4 & $1 \mathrm{~cm} * 1 \mathrm{~cm}$ lacerated wound in lower lip & 1 \\
\hline 5 & $3 \mathrm{~cm}$ sutured wound over right parietal scalp & 1 \\
\hline 6 & abrasion wound of size $3 * 2$ cms in left chin & 1 \\
\hline 7 & chest and pelvic injury & 1 \\
\hline 8 & chest injury and Facial Injury & 2 \\
\hline 9 & extremity injury & 1 \\
\hline 10 & head injury & 2 \\
\hline 11 & head injury with facial injury & 1 \\
\hline 12 & hollow viscus organ injury & 1 \\
\hline 13 & lower extremity injury & 1 \\
\hline 14 & No external injury & 54 \\
\hline
\end{tabular}

Maximum Extra Abdominal Solid Organ Injury were seen in No External Injury (54), followed by facial injury, chest and facial injury, head injury i.e. (02).

Table 4: Complications

\begin{tabular}{|c|c|c|}
\hline S. No. & Complications & No. \\
\hline 1 & Shock & 1 \\
\hline 2 & Acute renal failure & 1 \\
\hline 3 & ARF & 3 \\
\hline 4 & Bed Sore & 3 \\
\hline 5 & Burst Abdomen & 1 \\
\hline 6 & None & 81 \\
\hline 7 & Respiratory distress & 1 \\
\hline 8 & Septicemia & 3 \\
\hline 9 & Septicemia and shock & 1 \\
\hline 10 & Wound infection & 5 \\
\hline
\end{tabular}

Maximum Complications were seen in Wound infection (05), Septicemia (03), Bed Sore (03), ARF (03).
Table 5: Management of Blunt Trauma Abdomen According American Association for the Surgery of Trauma (AAST grading)

\begin{tabular}{|c|c|c|c|c|c|}
\hline \multirow[b]{2}{*}{$\begin{array}{c}\text { Abdominal Solid } \\
\text { Organ Injury }\end{array}$} & \multirow[b]{2}{*}{$\begin{array}{l}\text { No. of } \\
\text { Cases }\end{array}$} & \multicolumn{2}{|c|}{ Management } & \multicolumn{2}{|c|}{ Outcome } \\
\hline & & $\begin{array}{c}\text { conservativ } \\
\text { e }\end{array}$ & $\begin{array}{c}\text { operativ } \\
\text { e }\end{array}$ & Improved & $\begin{array}{c}\text { Not } \\
\text { Improved }\end{array}$ \\
\hline Liver & 57 & 46 & 11 & 52 & 05 \\
\hline Spleen & 44 & 27 & 17 & 39 & 05 \\
\hline Pancreases & 09 & 07 & 03 & 08 & 01 \\
\hline Kidney & 16 & 07 & 09 & 07 & 09 \\
\hline
\end{tabular}

Management of Blunt Trauma Abdomen highest cases were seen in Liver (57), Spleen (44), Kidney (16) \& Pancreases (09).

\section{Discussion}

Blunt trauma related Enteric Hollow visceral injury, by Jones et al. conducted for a period of 5 years took 2,045 patients in account, $70 \%$ patients were male, median age was 28 years, most common mechanism of injury was Road traffic accidents and total of $13 \%$ patients required Laparotomy ${ }^{[5]}$.

Blunt injury abdomen attending the tertiary hospital also demonstrated that males were the most commonly affected (75\%), most common age group was 21-30 years. the most common organ affected was the spleen.

Injury due to blunt trauma abdomen suggested that there should be a high index of suspicion for hollow viscus injury and management of patient changes on the basis of time of presentation, degree of contamination, associated injuries and general condition of patient. Study reported that the most common hollow viscus organ involved is Ileum $40 \%$, followed by Colon 25\%, Jejunum 20\%, Duodenum 10\% and Rectum 5\% [6].

Trauma is the second largest cause of disease accounting for $16 \%$ of global burden. This study took 71 patients in account and a retrospective analysis of management of patient was done. Most of the age group was 21-30 years and Male: Female 3.7:1 Motor vehicle accident was the most common cause of trauma. Most common extra abdominal injury was rib fracture. Spleen $(53 \%)$ was the most common organ involved in trauma. Clinical evaluation alone is inadequate as patients usually have altered mental status, initial assessment with eFAST and abdominal CT are very beneficial to detect abdominal injury ${ }^{[7]}$.

Study on abdominal trauma: patterns of injury, clinical presentation, organ involvement by Abdominal trauma is more common in age group of 21-40 years, male predominance (7.3:1), most common cause remains RTA (48\%), clinical features mostly are abdominal pain $(98 \%)$, tachycardia $(80 \%)$ and abdominal distension (50\%), most common organ injured is liver (32\%), spleen $(30 \%)$ and small bowel $(24.32 \%)$.

In retrospective group accidental cases were 49 i.e. $61.25 \%$ in prospective groups accidental cases were 105 i.e. $61.765 \%$. In retrospective group falling hard and blunt object over body comprises 13 , cases i.e. $7.6 \%$ in prospective groups assault by hard and blunt object comprises 5 cases i.e. $6.25 \%$. Most of the patients in our study mechanism of injury a retrospective analysis of 71 patients of BAT who were admitted in Kempegowda Institute of Medical Sciences hospital (KIMS, Bangalore, India) within a span of 18 months was done ${ }^{[8]}$.

\section{Conclusion}

In Blunt Trauma Abdomen injury there was maximum incidence of liver injury (57), followed by spleen injury (47), followed by renal injury (16) and followed by pancreatic injury cases (09) involved in BAT

Blunt Trauma Abdomen organ injury grading \& management 
according to AAST guideline (based on CT finding). In our study we tried to develop the management protocol for patients with clinical examination and findings.

\section{References}

1. Ameh EA, Nmadu PT. Gastrointestinal injuries from blunt abdominal trauma in children. 2004; 81(4):194-7.

2. Sule AZ, Kidmas AT, Awani K, Uba F, Misauno M. Gastrointestinal perforation following blunt abdominal trauma. 2007; 84(9):429-33.

3. Brown RA, Bass DH, Rode H, Millar AJ, Cywes S. Gastrointestinal tract perforation in children due to blunt abdominal trauma. 1992; 79(6):522-4.

4. TM Joseph. Blunt Injury of the Abdomen, 2015.

5. Jones EL, Stovall RT, Jones TS, Bensard DD, Burlew $\mathrm{CC}$, Johnson JL et al. Intra-abdominal injury following blunt trauma becomes clinically apparent within 9 hours. 2014; 76(4):1020-3.

6. Arikanoglu Z, Turkoglu A, Taskesen F, Ulger BV, Uslukaya O, Basol O, Aldemir. Factors affecting morbidity and mortality in hollow visceral injuries following blunt abdominal trauma. 14; 165(1):23-6.

7. Howes N, Walker T, Allorto NL, Oosthuizen GV, Clarke DL. Laparotomy for blunt abdominal trauma in a civilian trauma service. 2012; 29;50(2):30

8. Mehta N, Babu S, Venugopal K. An experience with blunt abdominal trauma: evaluation, management and outcome. 2014; 18; 4(2):599. doi: 10.4081/cp.2014.599. e Collection 2014. 